\title{
Diagnósticos, resultados e intervenções de enfermagem para crianças na Clínica Pediátrica de um hospital escola
}

\author{
Nursing diagnoses, outcomes and interventions for children in a \\ Pediatric Clinic of a school hospital \\ Diagnósticos, resultados y intervenciones de enfermería para niños en la \\ Clínica Pediátrica de un hospital de enseñanza
}

\begin{abstract}
Renata Valéria Nóbrega', Maria Miriam Lima da Nóbrega", Kenya de Lima Silva"I
I Secretaria Municipal de Saúde. Distrito Sanitário III.

Mestranda no Programa de Pós-Graduação em Enfermagem, CCS-UFPB. João Pessoa-PB, Brasil.

" Universidade Federal da Paraíba. Departamento de Enfermagem de Saúde Pública e Psiquiatria.

Programa de Pós-Graduação em Enfermagem. João Pessoa-PB, Brasil. Pesquisadora CNPq.

I'I Universidade Federal da Paraíba. Departamento de Enfermagem de Saúde Pública e Psiquiatria.

Hospital Universitário Lauro Wanderley. João Pessoa-PB, Brasil.
\end{abstract}

Submissão: 10/03/2010 Aprovação: 20/11/2010

\section{RESUMO}

Pesquisa exploratório-descritiva, desenvolvida com os objetivos de construir afirmativas de diagnósticos, resultados e intervenções de enfermagem para crianças internadas na Clínica Pediátrica de um hospital escola, tendo como base o Banco de Termos da Linguagem Especial de Enfermagem da referida Clínica Pediátrica e a CIPE ${ }^{\circledast}$ Versão 1.0; e de validar as afirmativas construídas com a participação de enfermeiros experts. Foram elaboradas 107 afirmativas de diagnósticos / resultados de enfermagem, das quais 48 atingiram IC $\geq 0.8$ (44,85\%) entre os enfermeiros experts, no processo de validação. Para estas 48 afirmativas, foram elaboradas 330 intervenções de enfermagem, das quais 270 alcançaram IC $\geq 0.8(81,81 \%)$ entre os enfermeiros experts. Acredita-se que a utilização dessas afirmativas poderá representar um relevante instrumento no processo de sistematização da assistência de enfermagem na Clínica Pediátrica do hospital escola.

Descritores: Enfermagem; Diagnósticos de Enfermagem; Criança hospitalizada; Enfermagem Pediátrica.

\section{ABSTRACT}

Exploratory-descriptive study, developed with the objectives of building diagnostic/ outcomes and nursing interventions statements, for children admitted to the Pediatric Clinic of a school hospital, based on the Bank of Terms of Nursing Special Language of that Pediatric Unit and, on ICNP ${ }^{\circledast}$ Version 1.0; and to validate those statements with the participation of expert nurses. It was assigned 107 nursing diagnoses / outcomes statements, of which 48 reached a $\mathrm{Cl} \geq 0.8(44.85 \%)$ among the expert nurses, in the validation process. For these 48 statements, it has been assigned 330 nursing interventions, of which 270 reached a $\mathrm{Cl} \geq 0.8(81,81 \%)$ among the expert nurses, in the validation process. It is believed that the use of these statements could represent an important tool in the process of systematization of nursing care in the Pediatric Clinic of the cited hospital school.

Key words: Nursing; Nursing diagnoses; Child, Hospitalized; Pediatric Nursing.

\section{RESUMEN}

Estudio exploratorio-descriptivo, desarrollado con los objetivos de construir declaraciones de diagnósticos / resultados y intervenciones de enfermería, para niños ingresados en la Clínica Pediátrica de un hospital escuela, con base en el Banco de Términos del Lenguaje Especial de Enfermería de dicha Unidad de Pediatría y con base en la CIPE ${ }^{\circledR}$ Versión 1.0 ; y validar las declaraciones con la participación de enfermeros peritos. Fueran asignadas 107 declaraciones de diagnósticos / resultados de enfermería, de las cuales 48 Ilegaron a un IC $\geq 0,8(44,85 \%)$ entre los enfermeros peritos, en el proceso de validación. Para estas 48 declaraciones, fueran asignadas 330 intervenciones de enfermería, de las cuales 270 Ilegaron a un IC $\geq 0,8$ $(81,81 \%)$ los enfermeros peritos, en el proceso de validación. Se cree que el uso de estas declaraciones podría ser un importante instrumento en el proceso de sistematización de la asistencia de enfermería en la Clínica Pediátrica del citado hospital escuela. Palabras clave: Enfermería; Diagnósticos de enfermería; Niño Hospitalizado; Enfermería pediátrica. 


\section{INTRODUÇÃO}

A Enfermagem é uma profissão antiga, que vem apresentando, ao longo da sua trajetória histórica, influências culturais de cada período, perpassando pelo saber das técnicas, princípios científicos e teorias. Passou a constituir-se em uma ciência moderna em meados do século XIX, a partir dos estudos de Florence Nightingale, quando se começou a sentir a necessidade de aprofundar o conhecimento específico, a fim de estabelecer seu papel profissional, bem como organizar e sistematizar o cuidado.

A Enfermagem é uma disciplina que se dedica, particularmente, à conservação da integridade, à reparação daquilo que constitui obstáculo à vida. Para focalizar esses obstáculos no ser humano, o profissional tem que apresentar um domínio e fazer uma busca constante do aprimoramento pessoal, uma vez que "os processos cuidativos de enfermagem envolvem características de presença genuína, de interação pessoal, de respeito ao outro, de empatia e afeto sob várias formas, todas aliadas à competência e habilidade"(1).

Em relação ao processo de desenvolvimento científico da Enfermagem, a década de 1950 foi destaque, quando as enfermeiras começaram a desenvolver modelos conceituais ou teorias de enfermagem, num esforço para identificar conceitos próprios e a sua utilização na prática. Nas décadas subsequentes, extensivas pesquisas foram realizadas, contribuindo para a introdução do processo de enfermagem nos Estados Unidos, na década de 1970, e, consequentemente, os sistemas de classificação ${ }^{(2)}$.

O processo de enfermagem representa um método sistemático e humanizado de prestação de cuidados, constituído de cinco passos: investigação, diagnóstico de enfermagem, planejamento, implementação ou intervenções de enfermagem, e avaliação ${ }^{(3)}$. O mesmo pode ser entendido como um trabalho profissional específico que pressupõe uma série de ações dinâmicas e inter-relacionadas para sua realização, ou seja, indica a adoção de um determinado método ou modo de fazer (Sistematização da Assistência de Enfermagem), fundamentado em um sistema de valores e crenças morais e no conhecimento técnico-científico da área ${ }^{(4)}$.

A segunda fase do processo de Enfermagem - diagnóstico de enfermagem é definida como uma ferramenta que possibilita individualizar o cuidado, transformar a prática da enfermagem, servir de base para as intervenções, organizar o saber da Enfermagem, introduzir o método científico na profissão, entre outras possibilidades. Tem como objetivo identificar os problemas existentes - os pontos fortes e essenciais -, obtidos a partir da análise das informações coletadas na investigação e essenciais para o planejamento do cuidado. Em síntese, elaborar um diagnóstico de enfermagem demanda análise e acurácia dos dados clínicos, permitindo ao profissional tomar decisões quanto aos resultados esperados ${ }^{(5)}$.

As diferentes fases do Processo de Enfermagem favoreceram o desenvolvimento de vários sistemas de classificação relacionados com os diagnósticos de enfermagem e, posteriormente, com as intervenções e com os resultados de enfermagem, modificando o modo de pensar, de falar e de atuar dos profissionais da enfermagem. A utilização desses sistemas na prática da profissão teve grande significado para o desenvolvimento da Enfermagem, estabelecendo padrões de cuidados que podem ser utilizados em qualquer parte do mundo, bem como permitir uma melhora na qualidade da assistência, por meio da sistematização, do registro e da quantificação do que os componentes da equipe de enfermagem produzem ${ }^{(6)}$.

Entre os vários sistemas de classificação desenvolvidos a partir das diferentes fases do processo de Enfermagem, destacam-se a Taxonomia da NANDA Internacional, a Classificação de Intervenções de Enfermagem (NIC), a Classificação de Resultados Esperados (NOC) e a Classificação Internacional para a Prática de Enfermagem (CIPE ${ }^{\circledR}$.

Neste estudo foi utilizada a $\mathrm{CIPE}^{\circledast}$ como sistema de classificação para a construção de afirmativas de diagnósticos, resultados e intervenções de enfermagem para crianças internadas na Clínica Pediátrica. A CIPE ${ }^{\circledR}$ está sendo desenvolvida pelo International Council of Nurses (ICN) (Conselho Internacional de Enfermagem - CIE), com o objetivo, sobretudo, de universalizar a linguagem de enfermagem. A CIPE $^{\oplus}$ Versão 1.0 é um recurso que pode ser utilizado para acomodar existentes vocabulários por meio de mapeamento cruzado para desenvolver novos vocabulários e para identificar relacionamentos entre conceitos e vocabulários. Além disso, permite aos enfermeiros documentar sistematicamente seus trabalhos realizados com clientes, famílias e comunidades usando diagnósticos, resultados e intervenções de enfermagem $^{(7)}$.

É relevante mencionar os trabalhos realizados no projeto intitulado "Identificação de dados essenciais de enfermagem para inserção em sistemas de informação: instrumental tecnológico para a prática profissional"(8), em que foram identificados os termos utilizados pelos componentes da equipe de enfermagem nos registros dos prontuários de pacientes das unidades clínicas do Hospital Universitário Lauro Wanderley (HULW/UFPB), e comparados com os termos da CIPE $^{\circledast}$, classificando-os como constantes e não constantes nesta classificação. Na Clínica Pediátrica foram identificados 289 termos, dos quais 168 (58,1\%) foram considerados como constantes na CIPE $^{\circledast}$ Versão 1.0 e 121 (41,9\%) como não constantes. Ao classificar os 168 termos constantes, utilizando o Modelo de Sete Eixos da CIPE ${ }^{\oplus}$ Versão 1.0, 61 estavam no eixo Foco; 3 no eixo Julgamento; 19 no eixo Meios; 37 no eixo Ações; 7 no eixo Tempo; 37 no eixo Localização; e 4 no eixo Cliente $^{9}$. Estes resultados levaram à construção do Banco de Termos da Linguagem Especial de Enfermagem da Clínica Pediátrica.

O presente estudo tem como objetivos desenvolver afirmativas de diagnósticos, resultados e intervenções de enfermagem para a Clínica Pediátrica de um hospital escola, tendo como base o Banco de Termos da Linguagem Especial de Enfermagem da Clínica Pediátrica e o Modelo de Sete Eixos da CIPE $^{\circledast}$ Versão 1.0; e validar as afirmativas de diagnósticos, resultados e intervenções de enfermagem desenvolvidas para a Clínica Pediátrica com a participação de enfermeiros experts que atuam na referida área. 


\section{METODOLOGIA}

Trata-se de uma pesquisa exploratório-descritiva, realizada na Clínica Pediátrica de um hospital escola da Universidade Federal da Paraíba, localizado na cidade de João Pessoa - Paraíba. Antes de sua realização, o projeto da pesquisa foi apreciado pelo Comitê de Ética em Pesquisa, do HULW/UFPB, em observância aos aspectos éticos preconizados na Resolução $n^{\circ} .196 / 96$ do Ministério da Saúde ${ }^{(10)}$, tendo obtido protocolo de aprovação $n^{\circ} 14 / 2008$.

$\mathrm{Na}$ etapa de construção das afirmativas de diagnósticos, resultados e intervenções de enfermagem, levou-se em consideração o Banco de Termos da Linguagem Especial de Enfermagem da Clínica Pediátrica e o Modelo de Sete Eixos da CIPE $^{\oplus}$ Versão 1.0. Na referida Clínica, foram desenvolvidas afirmativas de diagnósticos / resultados e intervenções de enfermagem, relacionadas às necessidades de crianças hospitalizadas para cuidados de saúde. Conforme recomendações do CIE, para composição das afirmativas de diagnósticos / resultados de enfermagem, foram utilizadas as seguintes diretrizes: incluir, obrigatoriamente, um termo do eixo Foco e um termo do eixo Julgamento; incluir termos adicionais, conforme a necessidade, dos eixos Foco e Julgamento e dos demais eixos. Para a construção de afirmativas relacionadas a intervenções de enfermagem, foram utilizadas as seguintes diretrizes: incluir, obrigatoriamente, um termo do eixo Ação e um termo Alvo, considerado como um termo de qualquer um dos demais eixos, com exceção do eixo Julgamento; podem ser incluídos termos adicionais, conforme a necessidade, dos outros eixos. Além disso, foi possível adicionar afirmativas de diagnósticos / resultados e intervenções de enfermagem identificadas na literatura da área e no Catálogo de diagnósticos / resultados de enfermagem da CIPE ${ }^{\oplus}$ Versão 1.1.

Após a construção das afirmativas, as mesmas foram submetidas a um processo de validação de conteúdo por especialistas da área, que concordaram em participar do estudo, assinando o Termo de Consentimento Livre e Esclarecido. Foram elaborados dois instrumentos: um para validação das afirmativas de Diagnósticos/ Resultados e outro para validação das Intervenções de Enfermagem, ambos para as crianças hospitalizadas na Clínica Pediátrica. Nestes instrumentos, foi solicitada a colaboração dos enfermeiros no sentido de apontar se as afirmativas eram aplicáveis à área da Clínica Pediátrica, e se eram utilizadas efetivamente na prática profissional. Em caso de discordância das afirmativas, requisitou-se, se possível, sugestões para sua adequação à realidade da prática de enfermagem.

Para o tratamento dos dados coletados na pesquisa, os instrumentos foram numerados e as variáveis contidas nos mesmos foram codificadas e inseridas em banco de dados, construído no programa Word for Windows. Os dados foram analisados utilizando-se estatísticas descritivas. Consideraram-se as afirmativas de diagnósticos / resultados e intervenções de enfermagem como validadas quando alcançaram um Índice de Concordância $(\mathrm{IC}) \geq 0,80$ entre os peritos participantes do estudo.

\section{RESULTADOS}

Seguindo os objetivos propostos e os critérios metodológicos, primeiramente foram elaboradas as afirmativas de diagnósticos / resultados de enfermagem. Nesta fase os termos constantes e não constantes, do Eixo Foco, presentes no Banco de Termos da Linguagem Especial de Enfermagem da Clínica Pediátrica foram selecionados e transferidos para um quadro do programa Microsoft Office Word 2007 for Windows. Em seguida, foram elaboradas 107 afirmativas de diagnósticos / resultados de enfermagem utilizando-se o Modelo de Sete Eixos da CIPE ${ }^{\oplus}$ Versão 1.0, a literatura da área e o Catálogo de Diagnósticos/Resultados de enfermagem da CIPE ${ }^{\oplus}$ Versão 1.1.

Após a elaboração das 107 afirmativas de diagnósticos / resultados, foi construído um instrumento e encaminhado para enfermeiros experts, considerados, neste estudo, como enfermeiros docentes e assistencias com mais de três anos de experiência na área de Enfermagem Pediátrica. Reconhece-se que selecionar experts para a validação de conteúdo é relevante e dificultoso ${ }^{(11)}$. Nessa etapa da pesquisa, participaram 12 enfermeiras, sendo cinco docentes da área de enfermagem pediátrica e sete enfermeiras assistenciais da Clínica Pediátrica. Neste estudo, coube aos experts confirmarem se as afirmativas diagnósticos / resultados de enfermagem identificam-se ou não com a sua prática clínica, de forma a aprovarem se as mesmas são reconhecidas pelas pessoas que exercem a assistência de enfermagem na Clínica Pediátrica.

Das 107 afirmativas de diagnósticos / resultados de enfermagem enviadas para as participantes da pesquisa, 48 obtiveram um IC $\geq 0,8(44,85 \%)$, sendo sugeridas três alterações, entre elas: Agitação leve e agitação moderada; passou a ser utilizado apenas um diagnóstico / resultado de enfermagem - Agitação (especificar: severa, moderada e leve); o diagnóstico / resultado de enfermagem Ingestão de líquidos diminuída passou a ser utilizado como Déficit de volume de líquidos. Dessa forma, passaram a ser consideradas 106 afirmativas de diagnósticos / resultados de enfermagem, entre validados e não validados.

Para a elaboração das Intervenções de Enfermagem foram utilizadas as afirmativas de diagnósticos / resultados de enfermagem validadas. Nesta etapa, utilizamos 46 diagnósticos / resultados de enfermagem, em virtude de dois (Incontinência intestinal e Ingestão alimentar deficiente) terem sido excluídos de forma não intencional pelas autoras quando da elaboração das afirmativas de intervenções de enfermagem.

As intervenções de enfermagem fazem parte do plano assistencial, o qual é traçado com o objetivo de eliminar ou reduzir um diagnóstico de enfermagem, buscando alcançar a meta ou o resultado preestabelecido ${ }^{(12)}$. Estas são consideradas estratégias utilizadas pelos enfermeiros para promover, manter e restaurar o estado de saúde do paciente, baseadas nos diagnósticos de enfermagem, para alcançar os resultados estabelecidos e solucionar as respostas humanas alteradas ${ }^{3}$.

Foram construídas 330 afirmativas de intervenções de enfermagem, distribuídas segundo os diagnósticos de enfermagem. Na fase de elaboração foram utilizadas as diretrizes do CIE, o Banco de Termos da Linguagem Especial de 
Enfermagem da Clínica Pediátrica e o Modelo de Sete Eixos da CIPE $^{\circledR}$ Versão 1.0, bem como o Catálogo de Intervenções de Enfermagem contido na CIPE ${ }^{\circledast}$ Versão 1.1, na busca de uma maior aproximação do verdadeiro perfil da clientela da Clínica Pediátrica. Em seguida, foi desenvolvido um instrumento com as intervenções de enfermagem para o processo de validação pelos enfermeiros experts. Nesta etapa da pesquisa participaram sete profissionais, sendo cinco enfermeiras assistenciais e dois docentes que atuam na referida clínica.

Das 107 afirmativas de diagnósticos de enfermagem e 330 intervenções de enfermagem elaboradas, 48 e 270 alcançaram IC $\geq 0,8$, respectivamente, conforme os quadros ilustrados abaixo (Quadros 1, 2 e 3) de acordo com alcance do IC em: IC $=1,0, I C=0.9, I C=0.8$.

\begin{tabular}{|c|c|}
\hline $\begin{array}{l}\text { DIAGNÓSTICO DE } \\
\text { ENFERMAGEM } \\
\text { IC }=1.0\end{array}$ & $\begin{array}{l}\text { INTERVENÇÕES DE ENFERMAGEM } \\
\qquad \text { IC } \geq 0.8\end{array}$ \\
\hline Comunicação prejudicada & $\begin{array}{l}\text { Ouvir atentamente a criança e/ou acompanhante; Estimular a comunicação; Identificar } \\
\text { barreiras à comunicação; Deixar que a criança expresse seus sentimentos e angústias; } \\
\text { Monitorar as mudanças no padrão da fala do paciente e no nível de orientação; } \\
\text { Proporcionar métodos alternativos de comunicação. }\end{array}$ \\
\hline Convulsão & $\begin{array}{l}\text { Colocar a criança em decúbito lateral para facilitar a drenagem da saliva; Observar e registrar } \\
\text { os sinais e sintomas na crise convulsiva; Aspirar às secreções da cavidade oral; Afastar objetos } \\
\text { possíveis de machucar a criança; Higienizar a criança se ocorrer liberação de esfíncter; } \\
\text { Proteger a cabeça da criança, colocando se necessário, travesseiro na cabeceira. }\end{array}$ \\
\hline Deglutição prejudicada & $\begin{array}{l}\text { Orientar a criança para mastigar bem os alimentos; Manter o ambiente tranquilo; Observar } \\
\text { se a criança tem regurgitação; Observar a deglutição e o esvaziamento da boca. }\end{array}$ \\
\hline Diarreia & $\begin{array}{l}\text { Investigar fatores causadores e/ou contribuintes da diarréia; Monitorar a pele perianal para } \\
\text { detectar irritações e úlceras; Monitorar as eliminações intestinais, inclusive frequência, } \\
\text { consistência, formato, volume e cor; Orientar a acompanhante sobre a importância da } \\
\text { dieta constipante; Estimular a ingestão de líquido. }\end{array}$ \\
\hline Dor & $\begin{array}{l}\text { Avaliar a dor quanto à localização, frequência e duração; Avaliar a eficácia das medidas } \\
\text { de controle da dor; Favorecer repouso/sono adequados para o alívio da dor; Investigar a } \\
\text { experiência de dor da criança; Preparar a criança para procedimento de administração } \\
\text { de medicamento; Ensinar o uso de técnicas não farmacológicas (relaxamento, imagem } \\
\text { orientada, musicoterapia, diversão, aplicação de compressas frias/quentes, aplicação de } \\
\text { massagem) antes, após e se possível durante a atividade dolorosa; Oferecer informações a } \\
\text { acompanhante sobre a dor, suas causas, tempo de duração, quando necessário. }\end{array}$ \\
\hline Febre & $\begin{array}{l}\text { Arejar o ambiente; Incentivar a ingestão de líquidos; Verificar a temperatura corporal } \\
\text { de } 4 \text { /4h; Remover o excesso de roupas; Promover conforto; Monitorar a ingestão e a } \\
\text { eliminação de líquidos; Observar reações de desorientação/confusão. }\end{array}$ \\
\hline Ferida infectada & $\begin{array}{l}\text { Avaliar a evolução da ferida; Observar e registrar secreção drenada; Monitorar a } \\
\text { temperatura; Orientar a criança e/ou acompanhante sobre os sinais e os sintomas de } \\
\text { infecção; Orientar criança e/ou acompanhante quanto à importância da higiene corporal; } \\
\text { Realizar curativo sempre que necessário; Avaliar realização de higiene pessoal pela } \\
\text { criança e/ou acompanhante; Utilizar técnicas assépticas. }\end{array}$ \\
\hline Hipotermia & $\begin{array}{l}\text { Avaliar a criança quanto aos sintomas associados - fadiga, fraqueza, confusão, apatia, } \\
\text { tremor; Ensinar a criança e/ou acompanhante os sinais precoces de alerta da hipotermia } \\
\text { - pele fria, palidez, vermelhidão; Evitar correntes de ar no ambiente; Manter a criança } \\
\text { aquecida com uso de cobertores; Manter a criança hidratada; Monitorar cor da criança; } \\
\text { Monitorar nível de consciência; Monitorar sinais vitais; Evitar infusões de líquidos gelados; } \\
\text { Monitorar o padrão respiratório; Avaliar risco de hipotermia. }\end{array}$ \\
\hline
\end{tabular}




\begin{tabular}{|c|l|}
\hline $\begin{array}{c}\text { DIAGNÓSTICO DE } \\
\text { ENFERMAGEM } \\
\text { IC = 1.0 }\end{array}$ & \multicolumn{1}{c|}{$\begin{array}{c}\text { INTERVENÇÕES DE ENFERMAGEM } \\
\text { IC } \geq \mathbf{0 . 8}\end{array}$} \\
\hline Peso adequado para idade & $\begin{array}{l}\text { Monitorar altura, peso, ingestão nutricional, estado cardiológico e pulmonar; Avaliar o nível } \\
\text { de desenvolvimento da criança; Orientar a acompanhante para fazer acompanhamento } \\
\text { mensal da criança até 1 ano de idade; Ficar atenta a sinais e sintomas de desenvolvimento } \\
\text { prejudicado da criança; Informar sobre os cuidados com a alimentação da criança. }\end{array}$ \\
\hline Recuperação adequada & $\begin{array}{l}\text { Observar e registrar os sinais e sintomas de melhora da criança; Enfatizar a criança e/ou } \\
\text { acompanhante quanto à participação no cuidado para promoção e recuperação de saúde; } \\
\text { Elogiar a criança e/ou acompanhante sobre a evolução do estado de saúde; Orientar a } \\
\text { criança e/ou acompanhante para manter os cuidados de higiene; }\end{array}$ \\
\hline Retenção urinária & $\begin{array}{l}\text { Monitorar a ingestão de líquidos; Medir o débito urinário; Orientar a criança e/ou } \\
\text { acompanhante sobre técnicas mencionais; Observar presença de edemas no corpo da } \\
\text { criança; Orientar quanto à ingestão de alimentos hipossódicos. }\end{array}$ \\
\hline
\end{tabular}

Quadro 1 - Afirmativas de diagnósticos / resultados de enfermagem (IC $=1.0)$ e de intervenções de enfermagem (IC $\geq 0.8)$ para crianças internadas na Clínica Pediátrica de um hospital escola. João Pessoa, 2009.

\begin{tabular}{|c|c|}
\hline $\begin{array}{l}\text { DIAGNÓSTICO DE } \\
\text { ENFERMAGEM } \\
\text { IC }=0.9\end{array}$ & $\begin{array}{l}\text { INTERVENÇÕES DE ENFERMAGEM } \\
\qquad I C \geq 0.8\end{array}$ \\
\hline Agitação & $\begin{array}{l}\text { Escutar atentamente, permitindo à criança e/ou acompanhante expressar sentimentos } \\
\text { verbalmente; Solicitar à criança e/ou acompanhante que defina quais tipos de atividades } \\
\text { promovem conforto e incentivá-los a realizá-las; Identificar e reduzir estressores } \\
\text { ambientais; Apoiar a criança e/ou o acompanhante quanto ao enfrentamento do } \\
\text { comportamento ansioso; Oferecer atividades de diversão voltadas à redução da tensão. }\end{array}$ \\
\hline Altura inadequada para idade & Avaliar o nível de crescimento e desenvolvimento da criança. \\
\hline Andar prejudicado & $\begin{array}{l}\text { Avaliar o progresso da criança na sua deambulação; Encorajar a deambulação } \\
\text { independente, dentro de limites seguros; Planejar as atividades da criança dentro do nível } \\
\text { de tolerância; }\end{array}$ \\
\hline $\begin{array}{l}\text { Angústia devido à ausência } \\
\text { da família }\end{array}$ & $\begin{array}{l}\text { Monitorar o estado emocional da criança; Oferecer ambiente calmo e agradável, para } \\
\text { proporcionar bem estar; Estabelecer relação de confiança com a criança; Proporcionar } \\
\text { oportunidade de a criança engajar-se nas atividades terapêuticas; Promover adaptação da } \\
\text { criança ao ambiente hospitalar. }\end{array}$ \\
\hline Apetite prejudicado & $\begin{array}{l}\text { Auxiliar a criança a se alimentar; Ensinar a acompanhante os conceitos de uma boa } \\
\text { nutrição para criança; Identificar problemas relacionados com a alimentação; Criar um } \\
\text { ambiente agradável e relaxante para as refeições; Orientar sobre a importância de a } \\
\text { dieta alimentar para a recuperação do estado de saúde; Planejar o atendimento para que } \\
\text { procedimentos desagradáveis ou dolorosos não ocorram antes das refeições; Encorajar a } \\
\text { criança a comer com outras refeições servidas na sala específica. }\end{array}$ \\
\hline $\begin{array}{l}\text { Capacidade para executar } \\
\text { auto-higiene }\end{array}$ & $\begin{array}{l}\text { Elogiar e estimular a criança e/ou acompanhante em relação à continuidade do } \\
\text { autocuidado com a higiene; Avaliar os cuidados de higiene; Avaliar autocuidado. }\end{array}$ \\
\hline $\begin{array}{l}\text { Falta de conhecimento sobre } \\
\text { a doença }\end{array}$ & $\begin{array}{l}\text { Estabelecer um relacionamento de confiança com a criança e acompanhante para facilitar a } \\
\text { aprendizagem; Orientar a criança e/ou acompanhante sobre o problema de saúde; Ensinar a } \\
\text { criança e/ou acompanhante as habilidades necessárias ao seu estilo de vida diário; }\end{array}$ \\
\hline
\end{tabular}




\begin{tabular}{|c|c|}
\hline $\begin{array}{l}\text { DIAGNÓSTICO DE } \\
\text { ENFERMAGEM } \\
\text { IC }=0.9\end{array}$ & $\begin{array}{l}\text { INTERVENÇÕES DE ENFERMAGEM } \\
\qquad I C \geq 0.8\end{array}$ \\
\hline Hipertermia & $\begin{array}{l}\text { Ensinar a criança e/ou acompanhante os sinais precoces de hipertermia; Orientar a } \\
\text { criança e/ou acompanhante quanto à importância de adequada ingestão de líquidos; } \\
\text { Arejar o ambiente; Promover o conforto para criança; Orientar a reposição de líquidos } \\
\text { após atividades com grandes gastos de energia; Verificar se as roupas ou cobertas não são } \\
\text { quentes demais para o ambiente ou para a atividade planejada. }\end{array}$ \\
\hline $\begin{array}{l}\text { Incapacidade para executar } \\
\text { auto-higiene }\end{array}$ & $\begin{array}{l}\text { Orientar a criança e/ou acompanhante quanto à escovação correta dos dentes e no enxágue } \\
\text { da boca, conforme a capacidade de autocuidado da criança; Encorajar a criança e/ou } \\
\text { acompanhante para continuação da rotina de higiene quando retornar a casa; Estimular hábitos } \\
\text { de higiene; Investigar as necessidades de aprendizagem da criança e/ou acompanhante; } \\
\text { Investigar se a criança tem material para higiene pessoal; Trocar roupas de cama diariamente; } \\
\text { Orientar a criança e/ou acompanhante quanto à lavagem do couro cabeludo, limpeza das } \\
\text { unhas e higiene íntima, conforme a capacidade de autocuidado da criança; Orientar sobre } \\
\text { importância dos hábitos de higiene; Higienizar cânula de traqueostomia. }\end{array}$ \\
\hline $\begin{array}{l}\text { Incontinência urinária } \\
\text { funcional }\end{array}$ & $\begin{array}{l}\text { Investigar se existem fatores contribuindo para a incontinência; Investigar déficits motores } \\
\text { - de mobilidade; Manter higiene íntima; Investigar déficits sensoriais cognitivos; Reduzir } \\
\text { as barreiras ambientais. }\end{array}$ \\
\hline Ingestão alimentar excessiva & $\begin{array}{l}\text { Incentivar reeducação alimentar; Estimular a criança a aderir à dieta alimentar; Oferecer } \\
\text { reforço positivo dos hábitos de dieta adequados; Orientar a criança a mastigar bem os } \\
\text { alimentos; Orientar a criança e/ou acompanhante sobre os riscos de saúde causados } \\
\text { pelo excesso de peso; Pesar a criança; Discutir com a criança e/ou acompanhante os } \\
\text { hábitos, os costumes e os fatores culturais que influenciam o peso; Encorajar a criança e/ } \\
\text { ou acompanhante na substituição de hábitos indesejáveis por hábitos favoráveis; Auxiliar a } \\
\text { criança na adaptação da dieta ao modo de vida e ao nível de atividade; Estimular autoestima. }\end{array}$ \\
\hline $\begin{array}{l}\text { Membrana da mucosa } \\
\text { hipocorada }\end{array}$ & $\begin{array}{l}\text { Verificar sinais vitais; Observar cianose; Estimular a hidratação; Observar sinais de } \\
\text { desidratação; Avaliar ingestão hídrica e alimentar. }\end{array}$ \\
\hline $\begin{array}{l}\text { Membrana da mucosa } \\
\text { normocorada }\end{array}$ & $\begin{array}{l}\text { Observar cianose; Observar sinais de desidratação; Observar coloração da mucosa; } \\
\text { Orientar criança e/ou acompanhante para manter os cuidados de higiene; Proporcionar } \\
\text { ambiente confortável. }\end{array}$ \\
\hline Náusea & $\begin{array}{l}\text { Explicar a criança e/ou acompanhante a causa e duração da náusea, caso seja conhecidas; } \\
\text { Orientar a criança e/ou acompanhante a evitar alimentos quentes ou frios; Limitar líquidos } \\
\text { durantes as refeições; Orientar a criança e/ou acompanhante para evitar deitar logo após } \\
\text { as refeições; Arejar o ambiente; Deixar a criança com roupas leves; Observar e registrar } \\
\text { fatores que contribuem para o aparecimento da náusea. }\end{array}$ \\
\hline Paralisia dos membros & $\begin{array}{l}\text { Prestar cuidados rigorosos para evitar agravamento do comprometimento da função } \\
\text { muscular; Orientar a criança e/ou acompanhante quanto à posição do membro afetado } \\
\text { para evitar agravamento da deformidade; Orientar quanto à mudança de decúbito para } \\
\text { evitar úlceras; Aplicar compressa quente para melhorar a função muscular; Orientar a } \\
\text { acompanhante para realizar higiene oral e corporal no leito; Manter o ambiente tranquilo. }\end{array}$ \\
\hline Peso inadequado para idade & $\begin{array}{c}\text { Pesar a criança diariamente; Investigar perda de peso, quantidade e período; Avaliar as } \\
\text { preferências alimentares; Averiguar a causa do peso inadequado; Discutir com o paciente } \\
\text { os hábitos, os costumes e os fatores culturais que influenciam o peso; Estimular higiene } \\
\text { oral antes e depois das refeições; Avaliar a aceitação da dieta. }\end{array}$ \\
\hline Pressão sanguínea controlada & Verificar pressão sanguínea. \\
\hline
\end{tabular}

Quadro 2 - Afirmativas de diagnósticos / resultados de enfermagem (IC $=0.9$ ) e de intervenções de enfermagem (IC $\geq 0.8$ ) para crianças internadas na Clínica Pediátrica de um hospital escola. João Pessoa, 2009. 


\begin{tabular}{|c|c|}
\hline $\begin{array}{l}\text { DIAGNÓSTICO DE } \\
\text { ENFERMAGEM } \\
\text { IC }=\mathbf{0 . 8}\end{array}$ & $\begin{array}{l}\text { INTERVENÇÕES DE ENFERMAGEM } \\
\text { IC } \geq \mathbf{0 . 8}\end{array}$ \\
\hline Cólica (especificar) & $\begin{array}{l}\text { Avaliar a cólica quanto à localização, frequência e duração; Avaliar a eficácia das } \\
\text { medidas de controle da cólica; Oferecer informações sobre as causas da cólica; } \\
\text { Monitorar a satisfação da criança com o controle da dor, a intervalos específicos. }\end{array}$ \\
\hline Déficit de volume de líquidos & $\begin{array}{l}\text { Avaliar resposta à infusão de líquidos; Controlar, rigorosamente, a terapia com } \\
\text { líquidos e eletrólitos; Estimular a ingestão hídrica; Pesar criança diariamente; Instruir a } \\
\text { acompanhante quanto à ingestão adequada de líquidos da criança; Medir débito urinário; } \\
\text { Avaliar a umidade da mucosa oral. }\end{array}$ \\
\hline Dificuldade para adormecer & $\begin{array}{l}\text { Monitorar o padrão de sono; Registrar o padrão do sono e quantidade de horas } \\
\text { dormidas; Identificar e reduzir estressores ambientais; Planejar as rotinas de cuidados } \\
\text { de enfermagem para que procedimentos desagradáveis ou dolorosos não ocorram após } \\
\text { as } 20 \text { h; Observar as circunstâncias físicas - apneia do sono, via aérea obstruída, dor/ } \\
\text { desconforto; Proporcionar um ambiente calmo e seguro; Orientar a acompanhante a } \\
\text { manter regularidade nos horários de deitar. }\end{array}$ \\
\hline Dor crônica & $\begin{array}{l}\text { Estabelecer metas para o controle da dor com a criança e a família e avaliá-las } \\
\text { regularmente; Investigar a experiência da dor usando a escala de investigação apropriada } \\
\text { ao desenvolvimento da criança e avaliando o seu comportamento; Promover um } \\
\text { ambiente confiável para a criança e acompanhante; Encorajar a criança a perceber que as } \\
\text { intervenções são tentativas de ajuda; Fazer com que a criança, a família e o enfermeiro } \\
\text { participem do controle da dor; Monitorar a dor após administração de medicamento. }\end{array}$ \\
\hline Dor epigástrica & $\begin{array}{l}\text { Registrar a ingestão/aceitação alimentar; Verificar posição e débito da sonda antes } \\
\text { de cada alimentação, se sondado; Registrar vômito quanto às suas características e } \\
\text { frequência; Ajudar o paciente a ficar em posições confortáveis; Monitorar a dor após } \\
\text { administração de medicamento; Avaliar a eficácia das medidas de controle da dor; } \\
\text { Descrever as características da dor, incluindo o início, duração, frequência, qualidade, } \\
\text { intensidade e os fatores precipitantes. }\end{array}$ \\
\hline Fadiga & $\begin{array}{l}\text { Manter o ambiente calmo e tranquilo; Explicar a acompanhante as causas da fadiga; } \\
\text { Identificar fatores que desencadeiam a fadiga; Orientar a acompanhante para deixar a } \\
\text { criança em repouso. }\end{array}$ \\
\hline Ferida limpa & $\begin{array}{l}\text { Avaliar o local da ferida cirúrgica; Orientar criança e/ou acompanhante quanto à } \\
\text { importância da higiene corporal; Orientar criança e/ou acompanhante quanto aos sinais } \\
\text { e sintomas de possível infecção; Avaliar o processo de cicatrização; Orientar criança e/ } \\
\text { ou acompanhante sobre cuidados com a ferida e cicatrização; Orientar criança e/ou } \\
\text { acompanhante quanto à lavagem das mãos antes e após o curativo da lesão; Utilizar } \\
\text { técnicas assépticas. }\end{array}$ \\
\hline Função renal prejudicada & $\begin{array}{l}\text { Controlar diurese das } 24 \text { horas quanto ao volume e características; Fazer controle } \\
\text { rigoroso de infusões venosas; Registrar ingestão e excretas de líquidos; Manter higiene } \\
\text { íntima; Avaliar a presença de edema no corpo, diariamente. }\end{array}$ \\
\hline Hidratação prejudicada & $\begin{array}{l}\text { Monitorar a ingestão; Monitorar as eliminações de líquidos; Monitorar os níveis de } \\
\text { eletrólitos séricos; Planejar uma meta de ingestão para cada oito horas; Investigar as } \\
\text { preferências da criança para ingestão de líquido. }\end{array}$ \\
\hline $\begin{array}{l}\text { Ingestão nutricional } \\
\text { prejudicada }\end{array}$ & $\begin{array}{l}\text { Ajudar a criança a alimentar-se; Informar a criança e/ou acompanhante quanto à } \\
\text { importância da nutrição para estabelecer o estado de saúde; Investigar problemas de } \\
\text { mastigação; Pesar a criança diariamente; Orientar criança e/ou acompanhante quanto } \\
\text { aos nutrientes necessários para recuperação do estado de saúde; Organizar o plano } \\
\text { de cuidados que evite odores nauseantes próximo das refeições; Oferecer pequenas } \\
\text { refeições frequentes; Manter um ambiente tranquilo. }\end{array}$ \\
\hline
\end{tabular}




\begin{tabular}{|c|c|}
\hline $\begin{array}{l}\text { DIAGNÓSTICO DE } \\
\text { ENFERMAGEM } \\
\text { IC }=\mathbf{0 . 8}\end{array}$ & $\begin{array}{l}\text { INTERVENÇÕES DE ENFERMAGEM } \\
\text { IC } \geq \mathbf{0 . 8}\end{array}$ \\
\hline $\begin{array}{l}\text { Integridade da pele } \\
\text { prejudicada }\end{array}$ & $\begin{array}{l}\text { Estimular a mudança de posição; Explicar cuidados com ostomias, pele e áreas } \\
\text { circunvizinhas; Manter a pele limpa e seca; Orientar higiene corporal e oral; Prevenir } \\
\text { lesões na pele; Proteger a pele contra infecção; Supervisionar o cuidado com a } \\
\text { pele; Cuidar do sítio de inserção de dispositivos invasivos; Educar a criança e/ou } \\
\text { acompanhante no cuidado cutâneo preventivo; Controlar a ingestão e a excreta - Balanço } \\
\text { hídrico; Estimular a hidratação; Realizar massagem na pele. }\end{array}$ \\
\hline $\begin{array}{l}\text { Mobilidade no leito } \\
\text { prejudicada }\end{array}$ & $\begin{array}{l}\text { Orientar a acompanhante para auxiliar a criança nos movimentos; Monitorar e registrar } \\
\text { diariamente qualquer sinal de complicação de imobilidade; Orientar a acompanhante } \\
\text { para a realização de atividade recreativa no leito; Realizar higiene corporal e do couro } \\
\text { cabeludo no leito. }\end{array}$ \\
\hline Pressão sanguínea alterada & $\begin{array}{l}\text { Monitorar presença de dispneia, fadiga; Orientar paciente/família sobre modificação de } \\
\text { fatores de risco (parar de fumar, dieta e exercícios) conforme apropriado; Supervisionar a } \\
\text { ingestão da dieta; Orientar quanto ao repouso; Monitorar a pressão sanguínea; Monitorar } \\
\text { o equilíbrio de líquido; Atentar para queixas de tonturas; Orientar períodos de repouso } \\
\text { frequente para maximizar a perfusão periférica; Avaliar cor, temperatura e textura de } \\
\text { pele; Avaliar monitoramento cardíaco; Orientar quanto aos exercícios moderados; } \\
\text { Orientar a criança e/ou acompanhante sobre a importância da comunicação a qualquer } \\
\text { desconforto torácico; Conscientizar a criança e/ou acompanhante sobre a importância da } \\
\text { redução do estresse; Identificar estado cardíaco. }\end{array}$ \\
\hline $\begin{array}{l}\text { Recuperação cirúrgica } \\
\text { retardada }\end{array}$ & $\begin{array}{l}\text { Observar e registrar sinais de infecção; Avaliar a ferida cirúrgica incluindo localização } \\
\text { e tamanho; Orientar a criança e/ou acompanhante o motivo do aumento de dias de } \\
\text { internação hospitalar. }\end{array}$ \\
\hline $\begin{array}{l}\text { Recuperação do estado de } \\
\text { saúde retardado }\end{array}$ & $\begin{array}{l}\text { Informar a criança e/ou acompanhante o motivo do retardo da recuperação do estado } \\
\text { de saúde; Enfatizar a importância da participação no cuidado para a promoção e } \\
\text { recuperação da sua saúde; Fornecer apoio e conforto, sem contestar, argumentar ou } \\
\text { desafiar a criança e/ou acompanhante com ilusões; Estabelecer metas junto com a } \\
\text { criança e/ou acompanhante para melhora de saúde da criança. }\end{array}$ \\
\hline Risco de sangramento & $\begin{array}{l}\text { Observar presença de manchas no corpo da criança; Orientar a acompanhante para } \\
\text { manter repouso; Orientar a acompanhante para realização de atividade recreativa no } \\
\text { leito; Orientar a criança e/ou acompanhante com a escovação dos dentes; Observar e } \\
\text { registrar presença de sangue. }\end{array}$ \\
\hline Sono prejudicado & $\begin{array}{l}\text { Auxiliar nas situações estressantes antes do horário de dormir; Auxiliar o paciente no } \\
\text { controle do sono diurno; Discutir com o paciente/família as medidas de conforto, } \\
\text { técnicas de monitoramento do sono e as mudanças no estilo de vida; Ensinar ao paciente } \\
\text { técnica de relaxamento; Encorajar uma rotina durante a noite facilitando a transição } \\
\text { do estado de alerta ao estado de sono; Observar as circunstâncias físicas - apneia do } \\
\text { sono, via aérea obstruída, dor/desconforto; Registrar e monitorar o padrão do sono e } \\
\text { quantidade de horas dormidas; Proporcionar um ambiente calmo e seguro. }\end{array}$ \\
\hline Volume de líquidos excessivo & $\begin{array}{l}\text { Fazer controle da ingestão de líquido; Observar e controlar gotejamento de infusão de } \\
\text { eletrólitos; Observar edemas; Avaliar resposta à infusão de líquido. }\end{array}$ \\
\hline
\end{tabular}

Quadro 3 - Afirmativas de diagnósticos / resultados de enfermagem (IC $=0.8$ ) e de intervenções de enfermagem (IC $\geq 0.8$ ) para crianças internadas na Clínica Pediátrica de um hospital escola. João Pessoa, 2009. 


\section{Discussão}

A estratégia de estabelecer uma padronização de cuidados individualizados a crianças hospitalizadas é uma ferramenta necessária para alicerçar a prática clínica do enfermeiro, pois subsidia tanto o estabelecimento das intervenções de enfermagem quanto à avaliação propriamente dita ${ }^{(13)}$. Além disso, o enfermeiro, ao planejar a assistência, garante sua responsabilidade junto à criança assistida, uma vez que o planejamento permite diagnosticar as necessidades do cliente, garante a prescrição adequada dos cuidados, orienta a supervisão do desempenho do pessoal, a avaliação dos resultados e da qualidade da assistência porque norteia as ações ${ }^{(14)}$.

No que diz respeito ao processo de trabalho do enfermeiro, a Sistematização da Assistência de Enfermagem impulsiona os enfermeiros a, continuamente, examinarem o que estão fazendo e estudarem como poderiam fazê-lo melhor. Dessa forma, permite o uso seguro do raciocínio clínico, observando de que forma esses diagnósticos se apresentam como instrumento qualificador da assistência ${ }^{(15)}$.

Das 107 afirmativas de diagnósticos / resultados de enfermagem construídas, 48 obtiveram um IC $\geq 0.8$ (44,85\%). No entanto, alguns dos 59 diagnósticos que foram apontados como não aplicáveis na Clínica Pediátrica são, seguramente, utilizados por alguns dos enfermeiros, mas não no quantitativo exigido para alcançar o IC preestabelecido. Ressaltam-se como exemplos os diagnósticos: Eliminação brônquica ineficaz ou prejudicada, Limpeza das vias aéreas prejudicada e Expectoração ineficaz; Continência intestinal e Defecação prejudicada; Eliminação urinária prejudicada, Padrão de eliminação prejudicado e Micção prejudicada; Integridade cutâneo-mucosa prejudicada; Padrão respiratório prejudicado e Respiração prejudicada.

Diante da situação supracitada, observou-se que vários diagnósticos não obtiveram IC $\geq 0.8$ devido à preferência das enfermeiras participantes por determinado vocabulário, apesar da utilização na prática assistencial. Mesmo assim, foi possível identificar que a CIPE ${ }^{\circledast}$ Versão 1.0 pode ser utilizada na Enfermagem Pediátrica, sendo validadas 48 afirmativas diagnósticas para a área.

A partir de então, surgem as inquietações das autoras quanto à percepção da importância dos diagnósticos referentes ao sistema respiratório, que não foram validados, embora se saiba que são muito utilizados na assistência a crianças hospitalizadas e pouco registrados na literatura da Enfermagem. Ressalta-se sobre o tema a escassez de trabalhos nesta clientela que contribuam para o manejo de ações preventivas ou corretivas sobre o problema das Infecções Respiratórias Agudas, sendo necessário identificar diagnósticos com vistas a fornecer indicadores para propor intervenções direcionadas e de competência exclusiva do enfermeiro, contribuindo para a elevação da qualidade da prática, através de um modelo de assistência mais eficaz ${ }^{(16)}$.

Em relação à afirmativa de intervenção de enfermagem, destaca-se "Verificar pressão sanguínea" para o diagnóstico "Pressão sanguínea controla" que apresentou IC $=1.0$, mas estudos da literatura mencionam que intervenções adequadas para esse diagnóstico são: continuar a dieta com baixa ingestão de sódio; realizar atividade física; Retirar alimentos gordurosos das refeições e lanches, entre outros.

Segundo a literatura, apenas nos últimos vinte e cinco anos o problema da hipertensão arterial recebeu a devida atenção na Pediatria. A incorporação dessa medida como parte do exame físico da criança, bem como a publicação de normas para a sua avaliação, possibilitou uma busca de intervenções para controle das elevações discretas da pressão arterial na infância ${ }^{(17)}$.

Diante dos fatores descritos sobre a intervenção acima, vale ressaltar que o desenvolvimento de construção de afirmativas de diagnósticos constitui um processo desafiador para facilitar a comunicação e a informação dos julgamentos de enfermeiros sobre as respostas dos seres humanos aos problemas de saúde e processos vitais ${ }^{(18)}$. Para tanto, evidencia-se a necessidade de testar e questionar se os diagnósticos e intervenções que utilizamos na sistematização do cuidado têm proporcionado ações articuladas para a qualidade da assistência.

Neste contexto, para a implantação e operacionalização do cuidar o enfermeiro necessita identificar a presença das necessidades humanas básicas afetadas nas crianças internadas e, assim, estabelecer os diagnósticos com as respectivas intervenções de enfermagem. Dessa forma, a equipe de enfermagem consegue prestar uma assistência planejada fundamentada em conhecimentos, viabilizando um cuidado objetivo e individualizado ${ }^{(19)}$.

É válido ressaltar que um processo constante de avaliação da implantação da SAE nas instituições se faz necessário na perspectiva do compromisso da avaliação da qualidade do processo de cuidado em saúde e das diversas formas de tratamento que vão surgindo. Precisamos, constantemente, nos questionar se os referenciais que utilizamos na sistematização do cuidado têm proporcionado ações de recuperação da saúde das crianças ${ }^{(20)}$.

O processo de validação das afirmativas finalizou com uma lista de 46 diagnósticos / resultados de enfermagem e de 270 intervenções de enfermagem. Vale ressaltar que algumas ainda necessitam de modificações para adequar-se à realidade da assistência na referida Clínica.

\section{CONSIDERAÇÕES FINAIS}

A elaboração de afirmativas de diagnósticos / resultados e intervenções de enfermagem é uma ferramenta relevante para a qualidade da sistematização da assistência, uma vez que o diagnóstico, quando utilizado corretamente, possibilita direcionar as ações por meio das intervenções a serem implementadas pela equipe de enfermagem. Além disso, possibilita a utilização de uma linguagem específica da área, garantindo uma comunicação clara, precisa e objetiva entre todos que compõem a equipe de enfermagem.

Vale ressaltar ainda que, no processo de validação das afirmativas de diagnósticos / resultados e intervenções de enfermagem, foram elencadas várias sugestões para modificações, relatadas na discussão. Por isso, recomenda-se dar continuidade ao estudo para que as afirmativas sejam reavaliadas por peritas e, consequentemente, se adaptem melhor às peculiaridades das crianças hospitalizas na Clínica Pediátrica. Recomenda-se também a utilização das diretrizes do CIE 
para a construção de Subconjuntos Terminológicos da CIPE , possibilitando uma integração do conhecimento científico ao conhecimento prático, assim como a utilização de uma linguagem específica da Enfermagem.
Espera-se que o uso das afirmativas de diagnósticos / resultados e intervenções de enfermagem no atendimento a crianças hospitalizadas seja eficaz e que possa proporcionar uma melhoria na qualidade da assistência a esta clientela.

\section{REFERÊNCIAS}

1. Cianciarullo TI, Gualda DMR, Melleiro MM, Anabuki MH. Sistema de Assistência de Enfermagem: evolução e tendências. 3ed. São Paulo (SP): Ícone, 2001.

2. Santos SMJ, Nóbrega MMLN. Ações de enfermagem identificadas no Projeto CIPESC e utilizadas no cuidado de pacientes com AIDS. Rev Esc Enferm USP 2004; 38(4):369-78.

3. Alfaro-Lefevre R. Aplicação do processo de enfermagem: promoção do cuidado colaborativo. 5ed. Porto Alegre (RS): Artmed; 2005.

4. Garcia TR, Nóbrega MML. Processo de enfermagem: da teoria à prática assistencial e de pesquisa. Esc Anna Nery Rev Enferm 2009 jan/mar; 13 (1):188-193.

5. Kruse MHL, Silva KS, Ribeiro RG, Fortes CV. Ordem como tarefa: a construção dos diagnósticos de enfermagem. Rev Bras Enferm 2008 mar./abr.; 61(2):262-266.

6. Nóbrega MML, Garcia TR. Perspectivas de incorporação da Classificação Internacional para a Prática de Enfermagem (CIPE ${ }^{\circledR}$ no Brasil. Rev Bras Enferm 2005 mar./abr.; 58(2):227-30.

7. Conselho Internacional de Enfermagem. Classificação Internacional para a Prática de Enfermagem $\mathrm{CIPE}^{\circledR}$ Versão 1.0. Versão oficial em Português. (tradutora: Heimar de Fátima Marin) São Paulo (SP): Algol Editora, 2007.

8. Nóbrega MML, Garcia TR. Identificação de dados essenciais de enfermagem para inserção em sistemas de informação: instrumental tecnológico para a prática profissional. [Relatório Técnico]. João Pessoa: DESPP/UFPB, 2008.

9. Lima MC. Banco de Termos da Linguagem Especial de Enfermagem da Clínica Pediátrica do Hospital Universitário Lauro Wanderley - UFPB. João Pessoa, 2008. 82 f. Monografia (Graduação em Enfermagem) - Universidade Federal da Paraíba.

10. Brasil. Ministério da Saúde. Conselho Nacional de Saúde. Comissão Nacional de Ética em Pesquisa - CONEP. Resolução $n^{\circ}$. 196/96: dispõe sobre pesquisa envolvendo seres humanos. Brasília (DF), 2005.

11. Galdeano LE, Rossi LA. Validação de conteúdo diagnóstico: critérios para seleção de expertos. Ciência, Cuidado e
Saúde 2006 jan./abr.; 5(1):60-66.

12. Silva KL. Construção e validação de instrumentos de coleta de dados para crianças de 0 - 5 anos. João Pessoa. 2004. 124p. Dissertação (mestrado) - Centro de Ciências da Saúde/ Universidade Federal da Paraíba, João Pessoa.

13. Chaves ECL, Carvalho EC, Rossi LA. Validação de diagnósticos de enfermagem: tipos, modelos e componentes validados. Rev. Eletr. Enf. [Internet] 2008; 10(2):513-520. Disponível em: < http://www.revistas.ufg.br/index.php/fen/ issue/view/740 $>$.

14. Andrade JS, Vieira MJ. Prática assistencial de enfermagem: problemas, perspectivas e necessidade de sistematização. Rev Bras Enferm 2005 maio/jun.; 58(3):261-5.

15. Yamamoto DM, Oliveira BRG, Viera CS, Collet N. O processo de trabalho dos enfermeiros em Unidades de Alojamento Conjunto pediátrico de instituições hospitalares públicas de ensino do Paraná. Texto Contexto Enferm [online] 2009; 18(2):224-232.

16. Monteiro FPM, Silva VM, Lopes MVO. Diagnósticos de enfermagem identificados em crianças com infecção respiratória aguda. Rev. Eletr. Enf. [Internet] 2006; 8(2):213-221. Disponível em: <http://www.fen.ufg.br/revista/revista8_2/ v8n2a06.htm $>$.

17. Salgado CM, Carvalhaes JTA. Hipertensão arterial na infância. J. Pediatr. (Rio J.) [online]. 2003; 79(suppl.1):S115-S124.

18. Santos ASR, Souza PA, Valle AMD, Cavalcanti ACD, Sá SPC, Santana RF. Caracterização dos diagnósticos de enfermagem identificados em prontuários de idosos: um estudo retrospectivo. Texto Contexto Enferm 2008 Jan./ Mar.; 17(1):141-9.

19. Bittencourt GKGD, Beserra PJF, Nóbrega MML. Assistência de enfermagem a paciente com Lupus eritematoso sistêmico utilizando a CIPE ${ }^{\circledast}$. Rev Gaúcha Enferm 2008 mar.; 29(1):26-32.

20. Castilho NC, Ribeiro PC, Chirelli MQ. A implementação da sistematização da assistência de enfermagem no serviço de saúde hospitalar do Brasil. Texto Contexto Enferm [online] 2009; 18(2):280-289. 